\title{
Artificial Intelligence in Global Epidemics, Part 1
}

\author{
Gurdeep Singh Hura ${ }^{1} \cdot$ Sven Groppe $^{2} \cdot$ Sarika Jain $^{3} \cdot$ Le Gruenwald $^{4}$
}

Published online: 9 November 2021

(c) Ohmsha, Ltd. and Springer Japan KK, part of Springer Nature 2021

\section{Introduction}

In the last year, most of the world's activities stopped due to the COVID-19 pandemic, but not research: we especially observed a fast race for new insights in COVID-19 and also in applying new technical approaches for COVID-19. In this sense, this special issue collects contributions about applying Artificial Intelligence (AI) to global epidemics.

In the fight against epidemics, medical staff is on the front line; however, behind the line, the battle is fought by the government officials, researchers, and data scientists. AI has been helping nations with computer modeling and simulation for predictions in many applications, such as predictions of the overall economic situation, tax incomes, and population development. In the same manner, AI can prepare the governments for emergency situations by backing the medical science. AI plays a key and cutting-edge role in preparing and dealing with the outbreak situation of global epidemics. It can help researchers analyze global data about known viruses to predict the information of the next pandemic and the impact it will have. Not only prediction, AI also plays an increasingly important role in assessing a country's readiness, early detection, identification of patients, generating recommendations, situation awareness, and more. It is up to the right input and the innovative ways by humans to leverage what AI can do.

Sven Groppe

groppe@ifis.uni-luebeck.de

Gurdeep Singh Hura

gurdeep.hura@gmail.com

Sarika Jain

jasarika@nitkkr.ac.in

Le Gruenwald

ggruenwald@ou.edu

1 University of Maryland Eastern Shore, Princess Anne, USA

2 University of Lübeck, Lübeck, Germany

3 National Institute of Technology, Kurukshetra, Haryana, India

4 The University of Oklahoma, Norman, USA 
As COVID-19 has grabbed the world and its economy today, an analysis of the COVID-19 outbreak and the global responses and analytics will pave the way in preparing humanity for such future situations. This special issue contains papers describing the role AI plays in various stages of a disease outbreak, with COVID-19 as a case study.

The special issue solicits submissions focusing on the perspectives and surveys of existing scenarios and consisting of research results in the following topics, but not limited to:

- Effects of COVID-19 Confinements

- Assessing Countries' Readiness for Coping with Epidemics

- Study related to Animal originated diseases

- Challenges in Battling with Epidemics

- AI for Forecasting

- $\mathrm{AI}$ and Detection

- Generating Recommendations

- $\mathrm{AI}$ in Genome Sequencing

- AI-assisted Testing

- Role of AI in Contact Tracing

- Situation Awareness

- Computational Drug Repurposing

\section{Review Process}

There was a great response to our call of this special issue: over 40 submissions is a strong sign that the COVID-19 outbreak is very much on the mind of many computer scientists. At the time of this writing, there are still submissions coming, which will be later added to subsequent issues of this journal. So far, 12 submissions have been accepted for the special issue, and some are still under review. This figure already shows that computer science research on COVID-19 is very competitive and only the best approaches are accepted by the scientific community.

\section{Selected Papers}

The selected papers are on many different topics. One paper applies fuzzy technologies [1]. Another introduces the AI technologies for COVID-19 containment [2]. Another publication proposes computer-aided-diagnosis as a service on decentralized medical cloud for efficient and rapid emergency response intelligence [3]. There are estimations of COVID-19 under-reporting in the Brazilian States [4] and methods to forecast COVID-19 outbreak [5, 6]. One publication deals with the chest X-ray image classification for COVID-19 early detection [7], while other approaches classify samples of ill and healthy persons according to the miRNA gene expression data [8]. Some authors propose an approach to support work-from-home driven by the COVID-19 pandemic [9], ensemble methods for heart disease prediction [10], a 
COVID19-Impact-on-Indian-Banking Ontology [11] and a context aware smart epidemic control framework [12].

We hope you find the selected papers interesting and informative. Have fun reading the papers!

\section{References}

1. Bharati, S.K.: An interval-valued intuitionistic hesitant fuzzy methodology and application. New Gener. Comput. 39, 377-407 (2021). https://doi.org/10.1007/s00354-021-00132-4

2. Surianarayanan, C., Chelliah, P.R.: Leveraging artificial intelligence (AI) capabilities for COVID-19 containment. New Gener. Comput. (2021). https://doi.org/10.1007/s00354-021-00128-0

3. Peyvandi, A., Majidi, B., Peyvandi, S., et al.: Computer-aided-diagnosis as a service on decentralized medical cloud for efficient and rapid emergency response intelligence. New Gener. Comput. (2021). https://doi.org/10.1007/s00354-021-00131-5

4. Paixão, B., Baroni, L., Pedroso, M., et al.: Estimation of COVID-19 under-reporting in the Brazilian states through SARI. New Gener. Comput. (2021). https://doi.org/10.1007/s00354-021-00125-3

5. Dash, S., Chakravarty, S., Mohanty, S.N., et al.: A deep learning method to forecast COVID-19 outbreak. New Gener. Comput. (2021). https://doi.org/10.1007/s00354-021-00129-Z

6. Sharma, N., Dev, J., Mangla, M., et al.: A heterogeneous ensemble forecasting model for disease prediction. New Gener. Comput. (2021). https://doi.org/10.1007/s00354-020-00119-7

7. Madaan, V., Roy, A., Gupta, C., et al.: XCOVNet: chest X-ray image classification for COVID-19 early detection using convolutional neural networks. New Gener. Comput. (2021). https://doi.org/10. 1007/s00354-021-00121-7

8. Marak, D.C.B., Halder, A., Kumar, A.: Semi-supervised ensemble learning for efficient cancer sample classification from miRNA gene expression data. New Gener. Comput. (2021). https://doi.org/ 10.1007/s00354-021-00123-5

9. Mandal, S., Khan, D.A., Jain, S.: Cloud-based zero trust access control policy: an approach to support work-from-home driven by COVID-19 pandemic. New Gener. Comput. (2021). https://doi.org/ 10.1007/s00354-021-00130-6

10. Karadeniz, T., Tokdemir, G., Mara, H.H.: Ensemble Methods for Heart Disease Prediction. New Gener. Comput. (2021). https://doi.org/10.1007/s00354-021-00124-4

11. Patel, A., Debnath, N.C., Mishra, A.K., et al.: Covid19-IBO: a Covid-19 impact on Indian banking ontology along with an efficient schema matching approach. New Gener. Comput. (2021). https:// doi.org/10.1007/s00354-021-00136-0

12. Gill, H.K., Sehgal, V.K., Verma, A.K.: CASE-CF: context aware smart epidemic control framework. New Gener. Comput. (2021). https://doi.org/10.1007/s00354-021-00135-1

Publisher's Note Springer Nature remains neutral with regard to jurisdictional claims in published maps and institutional affiliations. 